\title{
Erratum: Quantum kinetic equations for the ultrafast spin dynamics of excitons in diluted magnetic semiconductor quantum wells after optical excitation [Phys. Rev. B 95, 245203 (2017)]
}

F. Ungar, M. Cygorek, and V. M. Axt

(Received 5 December 2017; published 18 December 2017)

DOI: 10.1103/PhysRevB.96.239904

In Eq. (34b) there is a factor 2 missing in one of the terms proportional to $\delta\left(\omega_{x K}-\omega_{x_{1} K_{1}}\right)$. The correct equation should read

$$
\begin{aligned}
& \frac{\partial}{\partial t} \mathbf{s}_{x_{1} K_{1}}^{\perp}=\Gamma_{\mathbf{E}}^{\perp}+\frac{\pi I N_{\mathrm{Mn}}}{\hbar^{2} V^{2}} \sum_{x K}\left\{\delta ( \omega _ { x K } - \omega _ { x _ { 1 } K _ { 1 } } ) ( \mathbf { s } _ { x K } ^ { \perp } - \mathbf { s } _ { x _ { 1 } K _ { 1 } } ^ { \perp } ) \left[\left(2 J_{0}^{\mathrm{e}}-J_{s d}^{2} b^{\|}\right) F_{\eta_{\mathrm{h}} x x_{1}}^{\eta_{\mathrm{h}} K K_{1}}+\left(J_{p d}^{2} b^{\|}+2 J_{0}^{\mathrm{h}^{2}}-J_{p d} J_{0}^{\mathrm{h}} b^{0}\right) F_{\eta_{\mathrm{e}} x x_{1}}^{\eta_{\mathrm{e}} K K_{1}}\right.\right. \\
& \left.-\left(2 J_{p d} J_{0}^{\mathrm{e}} b^{0}+J_{p d} J_{0}^{\mathrm{h}} b^{0}-4 J_{0}^{\mathrm{e}} J_{0}^{\mathrm{h}}\right) F_{-\eta_{\mathrm{h}} x_{1}}^{\eta_{\mathrm{e}} K K_{1}}\right]-\left[\frac{b^{-}}{2} \delta\left\{\omega_{x K}-\left[\omega_{x_{1} K_{1}}+\left(\sigma_{\mathrm{e}}^{B} \omega_{\mathrm{e}}-\sigma_{\mathrm{Mn}}^{B} \omega_{\mathrm{Mn}}\right)\right]\right\}\right. \\
& \left.\left.+\frac{b^{+}}{2} \delta\left\{\omega_{x K}-\left[\omega_{x_{1} K_{1}}-\left(\sigma_{\mathrm{e}}^{B} \omega_{\mathrm{e}}-\sigma_{\mathrm{Mn}}^{B} \omega_{\mathrm{Mn}}\right)\right]\right\}+2 b^{\|} \delta\left(\omega_{x K}-\omega_{x_{1} K_{1}}\right)\right] J_{s d}^{2} F_{\eta_{\mathrm{h}} x x_{1}}^{\eta_{\mathrm{h}} K K_{1}} \mathbf{s}_{x_{1} K_{1}}^{\perp}\right\} \\
& +\left(\omega_{\mathrm{e}} \times \mathbf{s}_{x_{1} K_{1}}^{\perp}\right)\left\{1+\frac{1}{\omega_{\mathrm{e}}} \frac{I N_{\mathrm{Mn}}}{\hbar^{2} V^{2}} \sum_{x K}\left[\frac{J_{s d}}{\omega_{x K}-\omega_{x_{1} K_{1}}}\left[\left(2 J_{p d} b^{\|}-2 J_{0}^{\mathrm{h}} b^{0}\right) F_{-\eta_{\mathrm{h}} x x_{1}}^{\eta_{\mathrm{e}} K K_{1}}-2 J_{0}^{\mathrm{e}} b^{0} F_{\eta_{\mathrm{h}} x x_{1}}^{\eta_{\mathrm{h}} K K_{1}}\right]\right.\right. \\
& \left.\left.+\left(\frac{b^{+}}{\omega_{x K}-\left[\omega_{x_{1} K_{1}}-\left(\sigma_{\mathrm{e}}^{B} \omega_{\mathrm{e}}-\sigma_{\mathrm{Mn}}^{B} \omega_{\mathrm{Mn}}\right)\right]}-\frac{b^{-}}{\omega_{x K}-\left[\omega_{x_{1} K_{1}}+\left(\sigma_{\mathrm{e}}^{B} \omega_{\mathrm{e}}-\sigma_{\mathrm{Mn}}^{B} \omega_{\mathrm{Mn}}\right)\right]}\right) \frac{1}{2} J_{s d}^{2} F_{\eta_{\mathrm{h}} x x_{1}}^{\eta_{\mathrm{h}} K K_{1}}\right]\right\} .
\end{aligned}
$$

Since only the parallel case is discussed in the paper and the equation for the perpendicular spin density was given merely for the sake of completeness, all results and discussions throughout the paper remain unaffected.

Furthermore, there are some typographical errors in Eqs. (B1) in Appendix B. The correct equations should read

$$
\begin{aligned}
\frac{\partial}{\partial t} q_{\eta l x_{1} K_{1}}^{\uparrow / \downarrow x_{2}}= & -i\left(\omega_{x_{1} K_{1}} \pm \frac{1}{2} \omega_{\mathrm{e}}^{z}-\frac{1}{2} \omega_{\mathrm{h}}^{z}+\frac{I\left(J_{0}^{\mathrm{e}}+J_{0}^{\mathrm{h}}\right) N_{\mathrm{Mn}}}{\hbar V}\right) q_{\eta l x_{1} K_{1}}^{\uparrow / \downarrow x_{2}}-i \frac{1}{2} \omega_{\mathrm{e}}^{\mp} q_{\eta l x_{1} K_{1}}^{\downarrow / \uparrow x_{2}}+\epsilon_{i j l} \omega_{\mathrm{Mn}}^{i} q_{\eta j x_{1} K_{1}} \uparrow / \downarrow x_{2} \\
& -i \frac{I J_{s d}}{2 \hbar} F_{\substack{\eta \\
\eta x_{2} x_{1}}}^{-\eta_{\mathrm{h}} 0 K_{1}}\left( \pm\left\langle S^{l} S^{z}\right\rangle y_{x_{2}}^{\uparrow / \downarrow}+\left\langle S^{l} S^{\mp}\right\rangle y_{x_{2}}^{\downarrow / \uparrow}\right)+i \frac{I J_{p d}}{2 \hbar}\left\langle S^{l} S^{z}\right\rangle F_{\eta x_{2} x_{1} \eta_{\mathrm{e}} 0 K_{1}}^{\eta_{x_{2}}^{\uparrow} / \downarrow} \\
& -i \frac{I}{\hbar}\left\langle S^{l}\right\rangle\left(J_{0}^{\mathrm{e}} F_{\eta x_{2} x_{1}}^{-\eta_{\mathrm{h}} 0 K_{1}}+J_{0}^{\mathrm{h}} F_{\eta x_{2} x_{1}}^{\eta_{\mathrm{e}} 0 K_{1}}\right) y_{x_{2}}^{\uparrow / \downarrow}, \\
\frac{\partial}{\partial t} z_{\eta x_{1} K_{1}}^{\uparrow / \downarrow x_{2}}= & -i\left(\omega_{x_{1} K_{1}} \pm \frac{1}{2} \omega_{\mathrm{e}}^{z}-\frac{1}{2} \omega_{\mathrm{h}}^{z}+\frac{I\left(J_{0}^{\mathrm{e}}+J_{0}^{\mathrm{h}}\right) N_{\mathrm{Mn}}}{\hbar V}\right) z_{\eta x_{1} K_{1}}^{\uparrow / \downarrow x_{2}}-i \frac{1}{2} \omega_{\mathrm{e}}^{\mp} z_{\eta x_{1} K_{1}}^{\downarrow / \uparrow x_{2}} \\
& -i \frac{I J_{S d}}{2 \hbar} F_{\eta x_{2} x_{1}}^{-\eta_{\mathrm{h}} 0 K_{1}}\left( \pm\left\langle S^{z}\right\rangle y_{x_{2}}^{\uparrow / \downarrow}+\left\langle S^{\mp}\right\rangle y_{x_{2}}^{\downarrow / \uparrow}\right)+i \frac{I J_{p d}}{2 \hbar}\left\langle S^{z}\right\rangle F_{\eta x_{2} x_{1}}^{\eta_{\mathrm{e}} 0 K_{1}} y_{x_{2} \uparrow / \downarrow} \\
& -i \frac{I}{\hbar}\left(J_{0}^{\mathrm{e}} F_{\eta x_{2} x_{1}}^{-\eta_{\mathrm{h}} 0 K_{1}}+J_{0}^{\mathrm{h}} F_{\eta x_{2} x_{1}}^{\eta_{\mathrm{e}} 0 K_{1}}\right) y_{x_{2}}^{\uparrow / \downarrow} .
\end{aligned}
$$

The errors in the two equations above only occurred during preparation of the paper and are not present in the numerical evaluation of the equations. Thus, all numerical results of the paper have been obtained using the correct equations and remain unchanged. 